\title{
Tachycardia as "Shadow Play"
}

\author{
Andrey Moskalenko \\ The Institute of Mathematical Problems of Biology RAS \\ Russia
}

\section{Introduction}

Despite major scientific, medical and technological advances over the last few decades, a cure of tachycardia remains elusive. For example, the most of ventricular tachycardias (VT), including ventricular fibrillation, rank among life-threatening arrhythmias; but, in accordance with the recent multicenter investigations (CAST, ESVEM, CASCADE etc), treatment of the ventricular tachycardias using antiarrhythmic drugs of all classes leads to positive results in $58.5 \%$ of cases. In other words, the situation in its very essence indicates that the antiarrhythmic treatment is commonly prescribed nearly at random. In this context, investigation of ventricular arrhythmias must be continued in the most intensive manner, including the search for new diagnostically valuable features of cardiovascular signals.

Meanwhile, how odd it is that we should have taken the trouble of fighting tachycardia despite all the enormous progress in medicine knowledge! What we must understand is the plausible reason of this failure in treating tachycardia. The history of science gives us a lot of resembling examples convincing us that the actual state of affairs suggests a self-evident necessity of drastic alteration of the scientific paradigm that forms the basis of modern cardiology. Indeed, when all the enormous progress in modern diagnostic aids is accompanied by prescribing antiarrhythmic treatment nearly at random, we are made think seriously about adequacy of modern diagnostic aids. In other words, does modern diagnostics routine allow one to discern what should be discerned when choosing among cardiac remedy? Judging from the facts of our failure in treating tachycardia, we should conclude, that it is doubtful. Therefore, the difficulties that we encounter in our attempts to distinguish between different kinds of tachycardia correctly seem to remain to present day.

But what is the problem? We should bear in mind that the procedure of making a medical diagnosis, as "a general problem of function estimation based on empirical data" (Vapnik, 1999), consists of two tasks. The first one is creation of adequate classification, which should be free from fallacious distinction (a distinction without a difference) as well as from fallacious identification (assigning of two essentially different things to the same class of things based on a mere assumption or insufficient or erroneous grounds). To form classes (with or without the use of a priori reliable information on the statistical law underlying the problem) is the essence of the first part of making diagnosis, with the decision boundaries being constructed, that all the data can be separated into these classes. The second task of making diagnosis, in fact, consists in carrying out some diagnostic routine that allows one to assign an observed occurrence to one of the previously fixed classes. This bipartite procedure of making medical diagnosis is common for both human performance and performance of various learning machines in solving the problem of recognition. 
Investigation of how precisely modern diagnostic routines assign patients to one of the previously fixed classes of cardiac disorders, which are customary in the framework of a current conception, lies away from the subject of this chapter. Included among the results of this study were a number of examples that, in author's opinion, prejudice against adequacy of customary classification of tachycardia.

But in the beginning, it shows itself to the best advantage to recollect some of the basic principles of cardiology, making them be a subject of a small speculation.

\subsection{Can electrocardiography be omnipotent?}

For more than a century both classification and distinction of tachycardias have been grounded mainly on observation results of electrocardiography, the technique for recording the electrical activity of the heart. Clinical diagnostics in cardiology includes a lot of invasive as well as non-invasive procedures, but only few of them, such as electrocardiography, offers the possibility to characterize the dynamics of the cardiovascular system over time, from minutes to hours and days. Since being offered by Willem Einthoven, electrocardiography has been captivating doctors with its facility to register easily and objectively the events related to cardiac activity. Although later technological advances brought about better and more portable ECG devices, much of the terminology used in describing an ECG originated from Einthoven. This is just he who assigned the letters $\mathrm{P}, \mathrm{Q}$, $\mathrm{R}, \mathrm{S}$ and $\mathrm{T}$ to the various deflections, and described the electrocardiographic features of a number of cardiovascular disorders, which still remain in wide use. However the questions of what events exactly are registered, when recording ECG, and how exactly these events are related to functioning of the heart, recur time and again according to growing our knowledge of what exactly should been understood as functioning of the heart.

What do we observe indeed, when examining ECG? The theory implies (Titomir \& Kneppo, 1994) that even detailed electrocardiographic mapping avails to restore only the allocation of excitation process on surfaces of the heart, but not through the heart walls. Even the modern non-invasive electrocardiographic imaging (Ramanathan et al., 2004), ascending to the summit of electrodynamics, allows watching in real time the excitation process only on epicardium; nevertheless, there is need in many cases to know accurate excitation ways through the myocardium to provide heart patients with timely, accurate diagnoses. So, electrocardiography, the most engaging technique for observation of the heart, actually seems to be similar as if actor's personal character were guessed in shadow play flitted across a screen, does it not? Generally speaking, the ECG is a kind of shadowgram. Being in ignorance of this negative trait of the electrocardiography is resulting in a total misconception about examining ECG.

Inasmuch as physicians have been aspiring to knowing accurate excitation ways through the myocardium, the clinical cardiac electrophysiology began in the early 1970s with such a goal. And? Though electrophysiology laboratories improved both cardiac diagnostics and treatment, cardiac death remains a leading medical problem. However the cardiologic data were analysed, the results remained consistent: a cure of tachycardia requires improvement. Further investigations lead to the conviction that even very precise information about ways of excitation process in the myocardium is scanty for adequate diagnosis of cardiac disorders because, for example, cardiac disorders are likely to differ essentially in their etiology, while being similar in the ways of excitation spread. Hence, examining pictures of 
electrical activity of the heart obtained with electrocardiography, electrocardiographic imaging or electrophysiology mapping, doctors practise some sorts of divination and guesswork on the basis of shadow play nevertheless.

To improve the guess-work and to make it science-based mathematical approaches for ECG analysis were suggested in abundance. For example, some of the recent developments are described in (Loskutov \& Mironyuk, 2007; Wessel et al., 2007). In general, there is an apparent tendency for science to become more and more mathematical. Moreover, biochemical and even genetic methods have been recently united, with the other appropriate diagnostic tools to improve making precise diagnosis of tachycardia sort. For instance, a congenial form of the long-QT syndrome, LQT3, which is consistent with the clinical presentation of bradycardia-related arrhythmogenic episodes during sleep or relaxation, was detected by genetic methods (Noble, 2002a). It is noted (Crampin et al., 2004) that studies of human disease using microarray for gene expression are increasingly common in cardiovascular research and medicine. In spite of that, it is rather undoubtful that there are many thousands of genic combinations that can result (under one or another living conditions) in a phenotype with high risk of sudden cardiac death. Thus, cardiologists become more and more powerful in detailed description of the heart but can't seem to find a good solution for arrhythmic sudden death prediction and prevention.

All these facts lead us to the comprehension, that mechanisms of tachycardia are much more intricate than one used to consider them in the frames of the physiology of the 20th century. Drawing our analogy between electrocardiography and shadow pantomime still further, we ought to admit the possibility of quite objective evaluation of several actor's properties (such as, probably, their height or some other body traits) by observing their shadows flitted across a screen. The traditional electrocardiography keeps its positions in medicine since it gives likewise convenient possibility of swift and quite objective evaluation of several characteristics relative to cardiac performance. Some of medical questions can already be answered by visual inspection of the ECG paper strips themselves, e.g. whether the electrical excitation in different parts of the heart (sinus node, atrium, atrioventricular node, and ventricle) is normal or not. In some cases, location of arrhythmia origin as well as several morphologic disturbances, such as atrioventricular block, WPW syndrome, atrioventricular dissociation and injury currents after myocardial infarction can be visually identified on the ECG; however, this is nearly all reliable information which one should search, when using traditional examination of the ECG. "The ECG is, unfortunately, an unreliable indicator of potential arrhythmogenicity," a noted scientist explains the problem (Noble, 2002a). "Similar changes in form of the QT interval and T waveform can be induced by very different molecular and cellular effects, some benign, others dangerous." Other authors (Wessel et al., 2007) state very decidedly: "There exists no 'golden parameter' whose measurement is able to reliably describe and estimate all risks. From time to time there is an excessive enthusiasm of some scientists claiming that their new developed parameter is better than previously used ones. However, none of the known risk predictors probably is or can ever serve as an omnipotent outcome determinant." The last statement is referred to beat-to-beat analysis, but I am sure that the same is valid also for ECG analysis as a whole.

Thus, if ten years ago we did dare say about electrocardiography that there was "sufficient grounds to expect that the role of this method in differential diagnosis of cardiac arrhythmias will increase in future" (Moskalenko et al., 2001), now this thought appears to 
be rather doubtful. Admittedly, present-day mathematical approaches to analysis of time series (the ECG is a typical time series) gives birth to hopes to elicit information about intimate details of the functioning of the heart from the ECG. The mathematical approaches are considered bellow, but it is helpful to make more exact some details concerned with functioning of the heart before discussing these mathematical approaches.

\subsection{What should the activity of the heart and the action of the heart mean?}

Some time ago, I came up against the problem of appropriate translation of several basic ideas, which spread among the Russian scientists, into English. During my endeavours to solve it I realized that the puzzle lies far outside linguistic problems, but rather consists in essential difference of conceptual models of living creatures. Moreover, multitudinous literal adoption, when translating from English into Russian and vice versa, resulted with time in fanciful muddle of terms. One of such a pseudo-linguistic problems was discussed not long ago (Elkin \& Moskalenko, 2009) in connection with basic mechanisms of cardiac arrhythmias. In that case, the matter concerned fallacious use of the term "spiral wave" instead of "reverberator 1", which seems to be more correct when considering a twodimensional autowave vortex (for instance, a vortex of excitation in myocardium).

The terms such as "electrical function of the heart", "cardiac performance", "cardiac functioning" etc. are widely used in scientific literature although there are not any clear definitions of them. For the purpose of this preamble, it appears to be very helpful to emphasize the importance of distinguishing the phenomena that are associated with action of the heart 2, according to scientific and medical literature of Russia, from the phenomena that are reckoned among appearance of cardiac activity ${ }^{3}$. As a matter of fact, the Russian scientific doctrine is grounded, in many respects, upon the ideas that were evolved by P.K. Anokhin, P.V. Simonov, K.V. Sudakov and others who developed the theory of functional systems in the frames of cybernetic description of living things. Conceptually, the theory is close to control theory as well as to such relatively novel branches of science as biophysics, computational biology, and synergetics. In brief, the term "action" should be used to refer to events that can be characterised by target function in cybernetic way of description, while the term "activity" is appropriate for the other cases.

Hence, the terms "(any) cardiac activity" or "(any) activity of the heart" should be used only for designation of any aimless functioning of the heart. On the other hand, "the action of the heart" should be comprehended as functioning of the heart that is directed to maintenance of physiological homeostasis, which is the target function in this case. Obviously, the action of the heart can be put into effect only due to a quantity of control loops and guidance loop ${ }^{4}$,

\footnotetext{
${ }^{1}$ Some authors (Winfree, 1991) applied the term "rotor" to make distinction with "spiral wave", but the term is not in current use because of overload of its meaning. Both spiral wave and reverberator are sorts of re-entry, the main mechanism of tachycardia.

2 In Russian: "сердечная деятельность"; here translated as "action of the heart" in accordance with English-Russian Dictionary by Professor V.K. Mueller, 24-th Revised Edition; Moscow, "Russky Yazyk", 1995, p. 35 and p. 511.

${ }^{3}$ In Russian: “активность сердца"; “the electrical activity of the heart” 三“электрическая активность сердца".

${ }^{4}$ Notice that the control is used to maintain a desired output of the system under control, while the guidance is intended for shifting the system from a state A to a state B.
} 
which all together organize one and indivisible cardiovascular system. Electrical phenomena, that accompany the functioning of the heart and can be recorded by electrocardiography, are nowise satisfying the target of the cardiovascular functioning, because they are but side effects of the autowave function of the heart (Elkin \& Moskalenko, 2009). In English scientific and medical literature, the electrical phenomena to accompany the functioning of the heart are referred to as "the electrical activity of the heart" in a good accordance with the remarks above.

Though there are still some technical difficulties in distinguishing between these two concept nodes (aimed and aimless functioning) and though the language structures referred to them can be discussed as yet, nevertheless, it is very important for methodical purpose to keep in mind the necessity of such distinguishing. It is important because the distinguishing modifies a conceptual position of a cardiologist and can consequently change his or her evaluation of the symptoms. For example, the "new" cardiologic conception allows an assertion to make that the atrioventricular node reentrant tachycardia (AVNRT), which is a re-entrant rhythm within the atrioventricular (AV) node, should be considered in a number of cases as a variant of normal cardiac rhythm. Actually, the AVNRT is shown by recent investigations (Kurian et al., 2010) to be possible merely due to specific heterogeneity of AV node, which is peculiar to the human because of its genetic causes. But according to wellknown theory of biological evolution, the peculiarity would be eliminated ages and ages ago by mechanism of natural selection, if the heterogeneity of AV node were harmful for our ancestry. Nevertheless, we are very successful in observing the reverse: the hereditary trait was fixed by the natural selection. Hence, the supposition seems to be quite valid that the AVNRT was a variant of normal cardiac rhythm in that cases, when our remote ancestors required (for very quick running, for instance) developing the cardiac rhythm more rapid than the one that can be supplied by the sinoatrial node. Is it reasonable then to suppose that this hypothetic ancient mechanism of cardiac adaptation to extremely high load remains hitherto not to be rudimentary or atavistic, but plays still an important role as a potential mechanism of cardiac adaptation for human being? Since publishing this hypothesis (Elkin \& Moskalenko, 2009), some facts for its support have been revealed. For example, a very interesting case has been reported to me recently by professor Ardashev ${ }^{5}$ (in personal communication). At the time of scheduled medical examination of Russian Olympic team, the AVNRT was revealed in a racing cyclist during cardiac stress test. There were no patient complaints in this case, the cyclist felt himself very well and demonstrated remarkable results in the sport. Nevertheless, the objective data of electrocardiography were thought to be more important, and the man was operated to prevent a high risk of sudden cardiac death. There were no patient complaints after the ablation, this simple operation in our time, but the sportsman became incapable of sustaining his previous level of physical exertion and had to leave great sport. In my opinion, the case indicates how solicitous a cardiologist should be about such patients. Our adequate comprehension of cardiac functioning must be achieved more prompt, when doctors become more attentive to medical practice of this sort.

The case reported in the previous paragraph improves the conviction, that even the most solid data about the path of excitation spread in myocardium are just data about "shadow

5 Prof. Andrey V. Ardashev, M.D., Ph.D.; Director of Cardiac electrophysiology and arrhythmia service, 83 Clinical Hospital of Federal Biomedical Agency of Russia; official web-site: http://ardashevarrhythmia.ru/ 
play" ascertained exactly. The correct distinguishing of normal cardiac rhythm from pathological one, which appears to be similar visually and statistically, requires something greater than knowing the precise excitation path in the heart. We need something much more skilful than simple data-analysis of any existent type because the novel skilful dataanalysis must give us the ability to differ whether the functioning of the heart observed corresponds to the target function of the organism or conversely leads the biological system away from it. In the example above, the tachycardia seems to be a sort of adequate response of the organism to the requirements of its environment. To make adequate diagnosis, we need a complete model of situation, which consists of an adequate model of the patient and an adequate model of the surroundings in which he or she has resided for a sufficiently long period of time.

"The large complexity of cardiovascular regulation, with its multiplicity of hormonal, genetic and external interactions, requires a multivariate approach based on a combination of different linear and nonlinear parameters. $<\ldots>$ Biological control systems have multiple feedback loops and the dynamics result from the interplay between them. <...> Considering these rather system-theoretical characteristics, the development of nonlinear and also knowledge-based methods should lead to a diagnostic improvement in risk stratification. $<\ldots>$ A further aim, therefore, is, to go a qualitatively new step: the combination of data analysis and modeling" (Wessel et al., 2007). A cardiologist will be likely to gain the adequate diagnostic capacity of distinguishing the normal action of the heart from pathological one only, when having such a skilful knowledge-based model, realized either in computer or in the cardiologist's brain.

\subsection{Magic? Chaotic cardiology? Or cardiophysics?}

The discussion above reveals faultiness of the cardiologic paradigm, which is constructed owing to the epoch-making discoveries made by the greatest physiologist of the 19th and 20th centuries. The faultiness appears to be caused by our rather simple comprehension of how biological systems work, which arose from the historic specificity of scientific knowledge evolution. During 17-19th centuries the science development is known to be mainly grounded on the notions of determinism, with great advantage in applied mechanics to constitute their historical basis. It resulted in permeating the conception, which is referred to as mechanistic approach now, into many fields of science. Nor has medicine escaped the common lot, since all modern physiology is per se a manifestation of mechanistic approach in biology, as it was discussed formerly (Elkin \& Moskalenko, 2009). In accordance with scientific tradition, the phenomena observed in physiology are explained as a result of different mechanical movements. The movement of ions through membrane of a biological cell in order to explain the action potential is a good example of the approach. Prevalent endeavours of modern cardiologists to treat cardiac disorders by adjusting membrane channels using one or another drug is another such example.

But, there have been gathered a lot of observations by the end of the $20 \mathrm{~h}$ century that remain out the conceptual framework prescribed by the language of physiology. The time for new conceptual generalization has come. Physicists and mathematicians managed to find that processes to occur in "purely physical" systems (for example, in laser or even just in boiling water) are similar, in some sense, to those observed by physiologists in excitable biological 
tissues. Little by little, a new comprehension arose, that such phenomena, observed in biological tissues, as excitability, conductivity, all-or-nothing response, adiaphoria etc. are inherent not only to biological objects, but they widely happen in nonliving material as well. Notice that it is not a sort of drawing an analogy, but exactly a new valid generalization of scientific knowledge stored until now. The generalization entailed developing a new, more universal, language, namely the biophysical language. The new language enables not only rendering properly the description of all that was depicted before in the frames of physiology, but also representing, in unified terms, a wide range of experimental results which were hardly designated by the old language of physiologists.

The penetration of new scientific ideas in the old cardiology can be exemplified in developing the conception of autowave function of the heart (Elkin \& Moskalenko, 2009). Clear perception that autowave rather than electric properties of the heart give the base for the normal cardiac functioning seems to be really of great importance. Cardiac electrical activity, which was initially conceived by physiologists as the main cause of cardiac functioning, is rather similar to "shadows" of respective autowave processes. Physiologists used to think that the normal action of the heart is provided by conduction of ion channels of myocardial cells, but indeed it is provided by normal values of some integral myocardial parameters that characterize the heart as active medium. Different combinations of conduction of different ion channels can result in the same values of the integral parameters, and, therefore, some medical influence upon any sort of ion channels is likely to result in changing the integral myocardial parameters away from its values optimal for organism under given conditions. Further, cardiac abnormalities should be divided into two groups: those that are related with disorders of the cardiac autowave function and those that result from injuries of control and guidance loop in the integral cardiovascular system.

In addition to remarkable progress in biophysics, another important conceptual breakthrough of science was performed in the field that now is referred to as nonequilibrium thermodynamics, "physics of becoming" (Prigogine, 1980) or synergetics. "For centuries, humankind has believed that the world with all its form and structure was created by supernatural forces. In recent decades science has shaken these beliefs with the discovery of the exciting possibility of a self-created and self-creating world - of selforganization. Synergetics endeavours to reveal the intimate mechanisms of selforganization. The transitions from chaos to order, the nature of self-organization, the various approaches to it and certain philosophical inferences are outlined. Synergetics thus represents a remarkable confluence of many strands of thought, and has become a paradigm in modern culture" (Bushev, 1994). A significant role in this new conception pertains to the bifurcation theory as well as to the chaos theory. As to cardiology, there has been originated a modern discipline, computational biology, which applies the newest achievements of modern physics and mathematics. Note that all these modern studies are grounded, in many respects, on the dynamical systems theory, an area of applied mathematics used to describe the behaviour of complex dynamical systems. Much of modern research of dynamical systems is focused on the study of their chaotic behaviour. Despite initial insights in the first half of the 20th century, chaos theory became formalized as such only after midcentury, when it first became evident for some scientists that linear theory, the prevailing system theory at that time, simply could not explain the observed behaviour of certain experiments. Finite dimensional linear systems are well-known nowadays to be never chaotic; for a dynamical system to display chaotic behaviour, it has to be either nonlinear, or infinite-dimensional. Biological systems are complex and nonlinear, and, therefore, 
demonstrate complex and nonlinear behaviour, which may be chaotic, in many cases. The heart appears to be the same (Loskutov et al., 2009; Zhuchkova et al., 2009).

All these modern disciplines lead to the conclusion that often adequate comprehension of a complex system requires analysing not the observed values, but some of its integral characteristics, which can be mathematically obtained as a combination of a number of the observed. If speaking the language of mysticism ${ }^{6}$, the most important things occur on invisible plane supporting the visible one. In the dynamical systems theory, that invisible plane corresponds to phase space (state space) and parameter space of a dynamical system. Details of using the spaces were described elsewhere (Prigogine, 1980; Winfree, 1991; Loskutov et al., 2009). When a cardiologist attempts to analyse features of tachycardia observed on the ECG, the human mind appears to be unfit to observe the movement performed by the dynamical system corresponding to the patient under medical inspection. As a result, we have a very strange state of affairs: natural faculty of human mind does not allow correct diagnosis to be made in most of tachycardia cases. The results of analysing phase and parameter spaces of a patient seem only to give a good opportunity to clarify whether the cardiac phenomena observed correspond to the normal activity of the heart or some case of cardiac disorders happens.

Chaotic attractor is a good illustration of how important ideas of modern physics are for cardiology. Whether any sort of chaotic attractors correspond to the normal cardiac action, is a very good question.

Other crucial phenomena that must certainly be taken into account when treatment for a cardiologic patient is provided are caused by so-called "bifurcation memory", which has attracted special attention of investigators since recently (Feigin \& Kagan, 2004; Ataullakhanov et al., 2007; Moskalenko \& Elkin, 2009). The bifurcation memory is considered (Feigin \& Kagan, 2004) "within the framework of the general problem, when bifurcation situations generate in state space bifurcation tracks that isolate regions of unusual transition processes (phase spots)." Unusual behaviour of systems is attributed to the existence of the specific regions in phase space, the phase spots, where controllability of a dynamic system decreases dramatically. "Ships with high manoeuvring capabilities, aircraft and controlled underwater vehicles designed to be unstable in steady-state motion are dynamic systems of this class and are important for applications" (Feigin \& Kagan, 2004). Recent evidence suggests that similar phenomena can be found in the heart (Elkin \& Moskalenko, 2009; Moskalenko \& Elkin, 2009). The results of investigating the bifurcation memory in a cardiac model are presented and discussed below.

To gain a better insight into all these intricate things, the newest mathematical achievements as well as modern supercomputers should be widely adopted. "Mathematical modelling is widely accepted as an essential tool of analysis in the physical science and engineering, yet many are still sceptical about its role in biology. <...> It is however already clear that incorporation of cell models into tissue and organ models is capable of spectacular insights. $<\ldots>$ The potential of such simulations for teaching, drug discovery, device development,

\footnotetext{
${ }^{6}$ The point of Mysticism is that we humans have the capacity - a non-rational capacity - to identify with and to experience that invisible plane. It is possible, according to Mysticism, because in our deepest being, we belong to that invisible plane. Thus, a cardiologist probably uses such a non-rational capacity when guessing at the ECG, does he not?
} 
and, of course, for pure physiological insight is only beginning to be appreciated" (Noble, $2002 \mathrm{~b}$ ). Integrative physiology of the 21st century is set to become highly quantitative and, therefore, one of the most computer-intensive disciplines (Crampin et al., 2004; Noble, 2002a). Although, for historical reasons, the focus has been to study these events through experimental and clinical observations, mathematical modelling and simulation, which enable analysis at multiple time and spatial scales, have also complemented these efforts. "One of the intriguing opportunities presented by the availability of high resolution imaging and anatomically based computational models is that of the patient-specific modelling. That is, the generic model of the heart or lungs can be adjusted to match MR images of the heart or helical scan CT images of the lungs. Coupled with measurements of both gene sequence and physiological function for that individual, the realization of patientspecific, model-based clinical diagnosis becomes more feasible" (Crampin et al., 2004).

Thus, we ought to conclude that the 21st century seems to yield a new discipline, which is referred to as cardiovascular physics or cardiophysics (since it combines cardiology with novel achievements of physics). The cardiophysics is expected to solve the problems of prediction and prevention of sudden arrhythmic death.

\subsection{Polymorphic and monomorphic tachycardias: A distinction without differences?}

For about two last decades, it has been used to distinguish monomorphic and polymorphic ventricular tachycardias. The term "polymorphic" in cardiology refers to those ventricular arrhythmias that are characterised by the structure (morphology) of the QRS complexes continuously varied with time (Kukushkin et al., 1998). While a monomorphic ventricular tachycardia (MVT) is considered to distinguish itself by stable morphology of the QRS complexes in combination with constant value of the RR-intervals, a polymorphic one (PVT) is characterised by unstable morphology of the QRS; however, it remains still not fully clear how often and how profoundly the subsequent QRS complexes must be altered (Kukushkin $\&$ Medvinskii, 2004). Moreover, because electrocardiographic signals in PVT are of irregular shape, the very entity of PVT is not defined unambiguously in the known classifications of arrhythmias: its definition frequently overlaps with definitions of other types of cardiac rhythm disorders - see more elaborately in (Moskalenko et al., 2001). PVT is believed to precede ventricular fibrillation (VF) and sudden cardiac death. It has been stated (Kukushkin \& Medvinskii, 2004) that the stability of QRS morphology is reflected in the notion "degree of ECG polymorphism (variability)" and qualitatively corresponds to the extent of reentry nonstationarity. Discussion about etiology and mechanisms of PVT and VF remains after 160 years of inquiry due to exceedingly difficult methodological obstacles (Kukushkin \& Medvinskii, 2004; Kurian \& Efimov 2010). Despite this entire muddle, danger of arrhythmic death is linked by tradition with deepness of impression that polymorphic shape of the ECG creates in the mind of a cardiologist. Summarizing all these facts, we must conclude that some doubts are cast upon attempts to distinguish different types of cardiac disorders on base of practically identical set of signs. Should polymorphic shape of the ECG be apprehended as "shadow play", when essentially different cardiac disorders cast similar "shadows" on the ECG or when the same cardiac disorders appears on the ECG as noticeably different "shadows"? The remainder of the chapter is dedicated to studying the problem of how polymorphic shape of the ECG is significant for making adequate diagnosis. 


\section{Methods of the investigation: The Russian style in "affairs of the heart"}

Setting down quantitative criteria (Kukushkin et al., 1998) for identifying the electrocardiogram type correspondent to polymorphic shape of the ECG (polymorphic, monomorphic, or quasi-monomorphic ${ }^{7}$ ), which relied on the amplitudes and frequencies of the recorded signal, was likely the first attempt of accurate estimation of how polymorphic the shape of the ECG is. Mathematical approach for quantitatively assessing the amount of polymorphism present in electrocardiograms was proposed three years later (Moskalenko et al., 2001; Moskalenko et al., 2008). This approach was referred to in an earlier paper as normalized-value analysis of electrocardiographic variability (NVAEV, or "ANI-method" in Russian notation).

\subsection{The main idea of the ANI-method}

The ANI-method belongs to nonlinear and knowledge-based methods of time series analysis. Similar to a number of other methods of time series analysis (for example, Singular Spectrum Analysis, SSA), the first step of the ANI-method consists in transformation of the one-dimensional time series (the ECG in our case) into the trajectory matrix by means of a delay procedure. On the next step (instead of making the singular value decomposition, as it is in SSA), the normalized measure is calculated for each couple of columns of the trajectory matrix that is recognized as the nearest neighbours in some special sense. In such a way, a mathematical measure of "instant ECG variability" is obtained for each point of the source time series. Then some of average characteristics, so-called indices of ECG variability, are calculated in addition.

In the beginning, the new method was developed for automation of customary activities performed by a cardiologist assessing the ECG recorded during tachycardia in order to reckon the tachycardia among monomorphic or among polymorphic sets of an arrhythmia. To perform the assessing, one needs, in accordance with the known classifications of arrhythmias, to find adjacent arrhythmic cycles in the ECG (adjacent cycles of cardiac activation during arrhythmia), then to compare them in pairs, and finally to construct an assessment summarizing locally a few adjacent arrhythmic cycles. Thus, developers of such an automatic tool find themselves relatively free in their choice of key steps of the tool, namely: 1) a criterion for search, 2) a rule for the comparison procedure, and 3) a rule for the summarizing procedure.

In the course of designing the tool, we tried several ordinary approaches, and then an optimal combination of the key steps was empirically picked out. Of course, there is no good reason sufficient for insisting that the current realisation of the ANI-method is the most appropriate for solving the cardiologic problems which it is designed for. Therefore, any of the three key steps of the algorithm referred to as the ANI-method is likely to be replaced by a more appropriate one in order to improve the tool. Developing better

\footnotetext{
7 According to the authors (Kukushkin et al., 1998), arrhythmia is considered monomorphic if the changes in the frequency and the amplitude of the corresponding ECG is smaller then 15 and 30\%, respectively. Quasi-monomorphic is an arrhythmia that is "close to monomorphic". In other words, such a quasi-monomorphic arrhythmia is similar to a monomorphic one, being visually examined, but these two differ in a quantitative sense. The others are considered to be polymorphic.
} 
algorithms for analysis of electrocardiographic polymorphism may become a goal of future studies.

Anyway, the most important idea lying in the basis of the ANI-method is the decision to refuse flatly making any precise comparison between successive arrhythmic ventricular complexes on the ECG. Instead of precise comparison, an arbitrary ECG segment (reference sample) of length less than the length of the arrhythmic cycle is wittingly chosen as a pattern under consideration in order to be compared with the homologous phase segment of the successive (adjacent) arrhythmic ventricular complexes. The comparison must be reiterated with other pairs of such homologous segments while a statistically significant quantity of the comparison results is collected for each part of the ECG under study. The arbitrary ECG segment is also referred to as a sampling window (SaW), which width is taken equal $50-70 \%$ of the average arrhythmic cycle length. In practice, we chose the segment length (sampling window width) to be comparable with the shortest width of the QRS of the biological species under studying under control conditions (normal sinus rhythm), and the comparison is repeated for each point of the sampled ECG. In our experience, when errors of the comparison are present, their effects on the final result of quantitatively assessing polymorphic shape the ECG are often offset, at least in part. In other words, the fortuitous errors of such a comparison reciprocally compensate each other due to averaging the comparison results collected.

So, the new approach to analysis of arrhythmic ECG is free of the need to identify individual QRS complexes, because the ANI-method assesses the variability by numerically comparing adjacent segments of the ECG. The entire electrocardiogram or any of its fragments is described with two parameters, of which one is an index of ECG variability and the other specifies how this index changes with time. These two parameters are useful in assessing the arrhythmic polymorphism within one ECG and in comparing different electrocardiograms. Notice that, with the ANI-method for assessing the dynamics of changes in the ECG, there is no need to recognize individual peaks. Therefore, it is reasonable to expect that the variability indices will be useful in assessing real polymorphic electrocardiograms, especially when individual QRS complexes are difficult or impossible to identify.

After realising the current version of the ANI-method, some attempts to understand its mathematical sense were made. By this time, there are two variants of mathematical description given for the ANI-method. Both are adduced just below.

\subsubsection{Formalism by Medvinskii}

The first variant of mathematical description of the ANI-method was proposed by A. Medvinskii in a form appropriate for a discrete signal analysis (Moskalenko et al., 2001) and is given briefly here.

The procedure of comparison is implemented for each moment of time, which yields the local characteristic of electrocardiographic variability (instant ECG variability index $I_{i}$ ). This index is a tool for numerically comparing segment $i$ corresponding to the current position of the SaW with segment $j$, the first segment most closely resembling segment $i$ in the subsequent recording. The segment $j$ is supposed to be the homologous phase segment for the segment $i$. Formally, the procedure of their comparison is as follows. For any segment of the electrocardiogram, vector $F$ is defined: 


$$
F_{k}=\left(f_{k}, f_{k+h}, \ldots, f_{k+(p-1) h}\right),
$$

where $f_{n}$ is the signal amplitude at time $n$; $n$ varies from $k$ to $k+(p-1) / h$, where $p$ is the dimension of the so-called embedding space, and $h$ is the embedding step. The distance between the $i$ th and $j$ th vectors is determined by the norm:

$$
r_{i j}=\left|\mathbf{F}_{i}-\mathbf{F}_{j}\right| .
$$

Note that, for periodic signals, $r_{i j}=0$ if $|i-j|=m T$, where $m=0,1,2,3$, etc. For aperiodic signals, the smaller the difference between the $i$ th and the $j$ th segments, the smaller the $r_{i j}$.

Segments $i$ and $j$ are separated by a time interval $\tau_{i}: \tau_{i}=j-i, j>i$. Seeking the $j$ th segment and determining the $\tau_{i}$ value are based on the assumption that $j$ is a unique function of $i$. If $\tau_{i}$ were known, it would be possible to assess the similarity between the two segments using norm (1) in the $p$-dimensional embedding space. Therefore, we postulate:

$$
I_{i} \equiv \frac{1}{S_{i}} r_{i\left(i+\tau_{i}\right)}
$$

where $S_{i}$ is the peak-to-peak amplitude of the electrocardiographic signal in the sampling window corresponding to time $i$.

To automatically seek a segment most closely corresponding to the current SaW and separated from it by the shortest interval, we systematically scan an electrocardiogram segment of length $T_{s c W}$ (where $\mathrm{ScW}$ is an abbreviation for scanning window) using step size $h$. Every next segment within the scanning window is compared with the current sample by any technique (for example, a technique based on the use of an autocorrelation function or the norm in the embedding space). In simple cases in which the QRS complexes are identifiable, $\tau_{i}$ is equal to the inter-QRS interval, irrespective of the used technique. When the QRS complexes are difficult to identify, as in real polymorphic ventricular tachycardia, $\tau_{i}$ is formally set into correspondence with the position of the norm minimum. In other words, we assume that the $r_{i j}$ minimum determines the segment $j$ position in the scanning window. In this case, expression (2) reads

$$
I_{i}=\frac{1}{S_{i}} \min _{k \in(i, i+L)}\left(r_{i k}\right)
$$

This approach considerably saves the time for computing $I_{i}$ by combining the search for a segment homologous to the current sample with their comparison. The scanning window width $L$ is chosen to be as wide as the greatest width of normal QRS in the biological species under study.

For further analysis of electrocardiograms or their fragments of arbitrary lengths, it is useful to construct some integral characteristics of electrographic variability. Also, one of the simplest solutions is applied to construct the summarizing assessment. In our analysis, we use (i) the mean value of the $I_{i}$ function, or the electrocardiographic variability index $V_{1}$, and (ii) the coefficient of variation of the $I_{i}$ function, $V_{2}$. So, Index $V_{1}$ represents an average evaluation of the unlikeness of ECG segments inside the studied fragment and the index $V_{2}$ 
is its variation. To monitor the electrocardiogram changes with time, we calculate the variability index $V_{1 i}$ and the coefficient $V_{2 i}$ in some fixed-width window, (the averaging window, AW), corresponding to time $i$. Shifting the AW along the time axis, we obtain a trajectory in the $\left(V_{1 i}, V_{2 i}\right)$ parameter space, which enables one to visualize the detailed ECG dynamics. Note that the properties of the end segment of the ECG whose length is equal to the averaging window width are uncertain; therefore, the AW should not be too wide. On the basis of the definition of ventricular tachycardia, we chose the width of the averaging window to be six QRS widths. We assume everywhere that, unlike $V_{1 i}$ and $V_{2 i}, V_{1}$ and $V_{2}$ characterize segments that are considerably longer than the averaging window width.

\subsubsection{Formalism by Elkin}

An analytical form of mathematical description for the ANI-method was suggested by $\mathrm{Yu}$. Elkin in 2005 and is published here for the first time.

According to $\mathrm{Yu}$. Elkin, the ANI-method is a computational algorithm that could be analytically described as follows.

Let us define a function:

$$
R(t, T)=\left[\int_{t}^{t+T_{\text {Saw }}}(\varphi(\tau+T)-\varphi(\tau))^{2} d \tau\right]^{1 / 2},
$$

where $T_{\mathrm{SaW}}$, the width of the SaW, is a fixed value, which is a subject to adjustment (the $T_{\mathrm{SaW}}$ is taken equal $50-70 \%$ of the average arrhythmic cycle length, as it was mentioned above).

The procedure of seeking the next homologous phase segments is executed within the scanning window, $\mathrm{ScW}$, with its width confined to an area of expectation to find the adjacent arrhythmic cycle. At each instant of time $t$, a segment is supposed to be the adjacent homologous phase segment for the current sampling window, SaW, if the segment is situated at the distance $T_{0}(t)$ such, that

$$
R\left(t, T_{0}(t)\right)=\min _{T_{S a W} \leq T \leq T_{S c W}}(R(t, T))
$$

For the ECG under consideration, the comparison of the homologous phase segments is repeated continuously with some constant time step $\Delta t_{0}$, without binding to any phase of an arrhythmic ventricular complex. The comparison of the pair recognized to be the adjacent homologous phase segment is carried out by the formula:

$$
I(t) \equiv \frac{1}{S(t)} R\left(t, T_{0}(t)\right)
$$

The function $T_{0}(t)$ is referred to as quasi-period, so long as, for a cyclic process, it corresponds to a quantity that is analogous to the period of a periodic process; for a periodic signal, $T_{0}(t)$ is exactly equal to its period.

Further, the ECG variability indices $V_{1}$ and $V_{2}$ can be produced for any fragment of $I(t)$. The functions $V_{1}(t)$ and $V_{2}(t)$ can be yielded with the help of shifting the AW along the time axis. 


\subsubsection{ANI -2003}

It is noteworthy that the current implementation of the NVAEV (Medvinsky et al., 2003), referred to as ANI-2003, is improved by preliminary normalization of time series within the $\mathrm{ScW}$, in order to increase the stability of the algorithm. The normalization is performed by substituting the sample mean or sample deviation into the formula for standard score. All results presented bellow are obtained with use of the ANI-2003.

\section{Results and discussion}

We studied pseudo electrocardiograms (pseudo-ECGs) obtained from in vitro studies of ventricular arrhythmias ("physiological ECG") as well as from numerical simulations ("numerical ECG").

Each "physiological ECG" represented a time series constructed by weighted summation of separate electrocardiograms obtained by multielectrode mapping of excitation propagation during an arrhythmia obtained from the isolated wall of the ground squirrel right ventricle. The experimental model and the procedure for constructing the pseudo-ECG were described in detail elsewhere (Sarancha et al., 1997; Kukushkin et al., 1998).

For calculation of "numerical ECGs" we used the Aliev-Panfilov mathematical model of heart tissue (Aliev \& Panfilov, 1996):

$$
\begin{gathered}
\frac{\partial u}{\partial t}=\Delta u-k u(u-a)(u-1)-u v \\
\frac{\partial v}{\partial t}=\varepsilon(u, v)(-u-k u(u-a-1)) \\
\varepsilon(u, v)=\varepsilon_{0}+\frac{\mu_{1} v}{u+\mu_{2}} .
\end{gathered}
$$

The parameters in the equations were adjusted (Aliev \& Panfilov, 1996) to accurately reflect cardiac tissue properties $\left(k=8.0 ; \mu_{1}=0.2 ; \mu_{2}=0.2 ; a=0.15 ; \varepsilon=0.01\right)$. In our simulations, the parameter $\mu_{2}$ was equal to 0.3 or 1.3 , with the parameter $a$ being varied from 0.12 to 0.19 . Note that the parameter $a$ specifies the threshold of excitation. The simulations were carried out in 2D excitable media (128 elements along each dimension) with von Neumann boundary conditions. The details of the simulations and the procedure for constructing the pseudo-ECGs in the simulations were described in detail elsewhere (Moskalenko \& Elkin, 2007; Moskalenko \& Elkin, 2009).

\subsection{Tachycardia of the lacetic type}

The reverberator, a typical autowave phenomenon in two-dimensional excitable media, can be described simplistically as a half of a plane wave curved around its break point. The break point is also called the tip of the reverberator. Reverberator behaviour is commonly sketched in the terms of movement of its tip. Autowave reverberators are known to be the main cause of different kinds of tachycardia. As long as the dynamics of the reverberators rides on myocardium state, the myocardium state can be estimated, perhaps, by details of their dynamics. 
Formerly, three types of reverberator tip movement in homogeneous two-dimensional medium were known. These are 1) uniform circular movement, 2) meander, i.e. twoperiodic movement, with the tip moving along a curve similar to cycloid (epicycloid or hypocycloid), and 3) hyper-meander, i.e. a 'complex' or maybe 'chaotic' movement whose wave tip trajectory could not be described in terms of two periods. Recently we found (Moskalenko \& Elkin, 2009) a new autowave behaviour, which we called the lacet, namely, the transformation of reverberator motion from a two-periodic meander into oneperiodic circular rotation due to spontaneous deceleration of reverberator drift (Fig. 1). The lacet is perhaps a phenomenon of so called bifurcation memory.

A

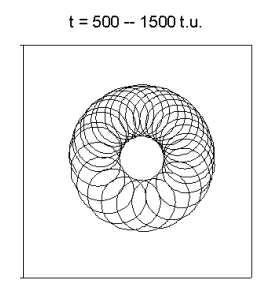

$t=1500-3500$ t.u

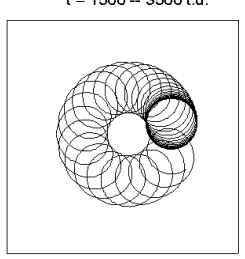

B
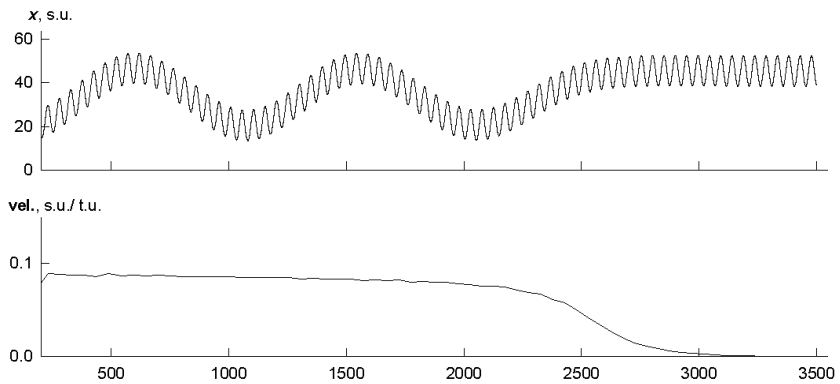

C ECG
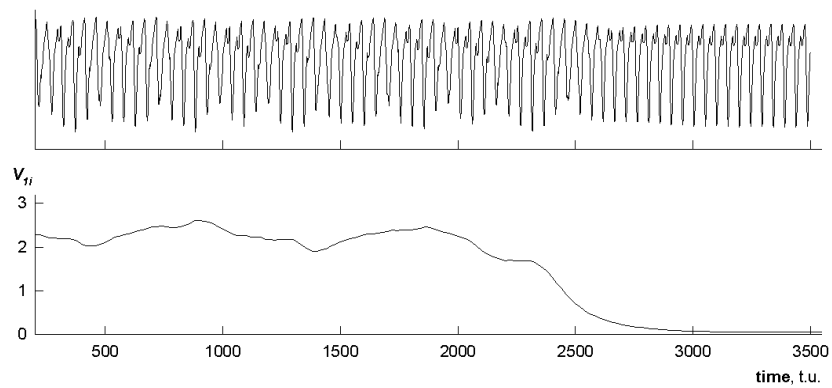

Fig. 1. The transition from polymorphic to monomorphic tachycardia of the lacetic type in the Aliev-Panfilov model at $\mathrm{a}=0.1803$. A. The trajectory of the reverberator tip in the case of the vortex drift deceleration (the lacet type of the vortex motion). For the convenience, the trajectory is segregated into two pictures because some different parts of the trajectory overlap. B-C. From top to bottom: the dynamics of the $x$ coordinate of the reverberator tip, the dynamics of velocity of the instant centre of the autowave vortex, the ECG, and index of the ECG variability, $V_{1}(t)$. All the graphs have the same horizontal scale.

It was demonstrated in computational simulation (Moskalenko \& Elkin, 2007; Elkin \& Moskalenko, 2009) that the lacet in the myocardium coincides with spontaneous transition from polymorphic to monomorphic arrhythmia in the ECG dynamics. Also, it was shown by the example of the lacet (Moskalenko, 2010) that the information revealed in ECG with the ANI-2003 corresponds sufficiently to velocity of the reverberator drift. Fig. 1 represents these results. The comparison of the velocity of reverberator drift and ECG dynamics described with $V_{1}(t)$ shows that there is perfect coincidence between them. 
By visual inspection of the ECG paper strips, there is no way to distinguish a tachycardia caused by an ordinary autowave vortex from a tachycardia of the lacetic type. Observing the ECG on Fig. 1 during but the first 2000 time units, cardiologist will certainly think it to be just typical re-entrant tachycardia, because there is no obvious difference between ECG shapes of these different sorts of tachycardia. Moreover, even the transition from polymorphic to monomorphic type of tachycardia, being spontaneous, appears to be similar to the transition caused by anchoring the autowave vortex in an obstacle (cardiac veins, for instance). But as a matter of fact, the dynamic system, the heart, is in the state of bifurcation memory, and, consequently, therapeutic treatment in this case is likely to result in unpredictable effect, since controllability of the system has decreased dramatically.

Thus, the result demonstrates that two different types of tachycardia produce similar "shadows" on the ECG. Therefore, more sophisticated methods to analyse cardiac activities are required so that the adequate treatment could be prescribed.

\subsection{Is monomorphic tachycardia indeed monomorphic?}

The dependence of some ECG characteristics on the excitation threshold was studied by mathematical modelling of cardiac arrhythmia in a 2D homogeneous excitable medium (Moskalenko \& Elkin, 2007). The authors of the model used here (Aliev \& Panfilov, 1996) state that normal excitability corresponds to $a=0.150$. In the present work we have observed monomorphic tachycardia at this parameter value as well as at those higher and lower. In other words, monomorphic tachycardias can arise both at elevated and at lowered excitability (Fig. 2).

This result appears to be very important in the medical aspect. It indicates that re-entrant monomorphic ventricular tachycardia may require different treatment depending on the sign of the deviation of excitability from the norm. Currently, physicians do not attempt to distinguish such cases. If further studies prove that these results can be extended to clinical ECGs, the more accurate diagnosis would make possible more expedient therapy.

For monomorphic re-entrant tachycardias, indices $V_{1 i}$ and $V_{2 i}$ also behave unexpectedly. Comparing the cases of monomorphic pseudo-ECGs in Figs. 3, one can find that the trajectories of the "physiological ECGs" and the trajectories of the "numerical ECGs" have significantly different locations, with the ECGs shape having no evident visual differences. It is interesting that the "numerical ECGs" are more "deterministic" than the "physiological ECGs" in monomorphic cases. We explain these results in the following manner. In both our natural and numerical experiments, monomorphic arrhythmias were caused by a $2 \mathrm{D}$ autowave vortex. Therefore, it is reasonable that the "numerical ECGs" have no stochastic component and are estimated to be more regular. However, the difference may also be supposed to result from more chaotic behaviour of real myocardium. A further study could assess the participation of stochastic and chaotic components in producing the difference.

Note, however, that changes in $V_{1}$ are usually correlated fairly well with changes in $V_{2}$ when it related to polymorphic "physiological ECGs", but there is no correlation observed in the "numerical ECGs" Research into the nature of this fact will shed new light on the mechanisms of polymorphic tachycardias. It is also important to address the issue of whether the changes in the myocardial excitation patterns are correlated with the changes in the trajectories in the $\left(V_{1 i}, V_{2 i}\right)$ parameter plane. 


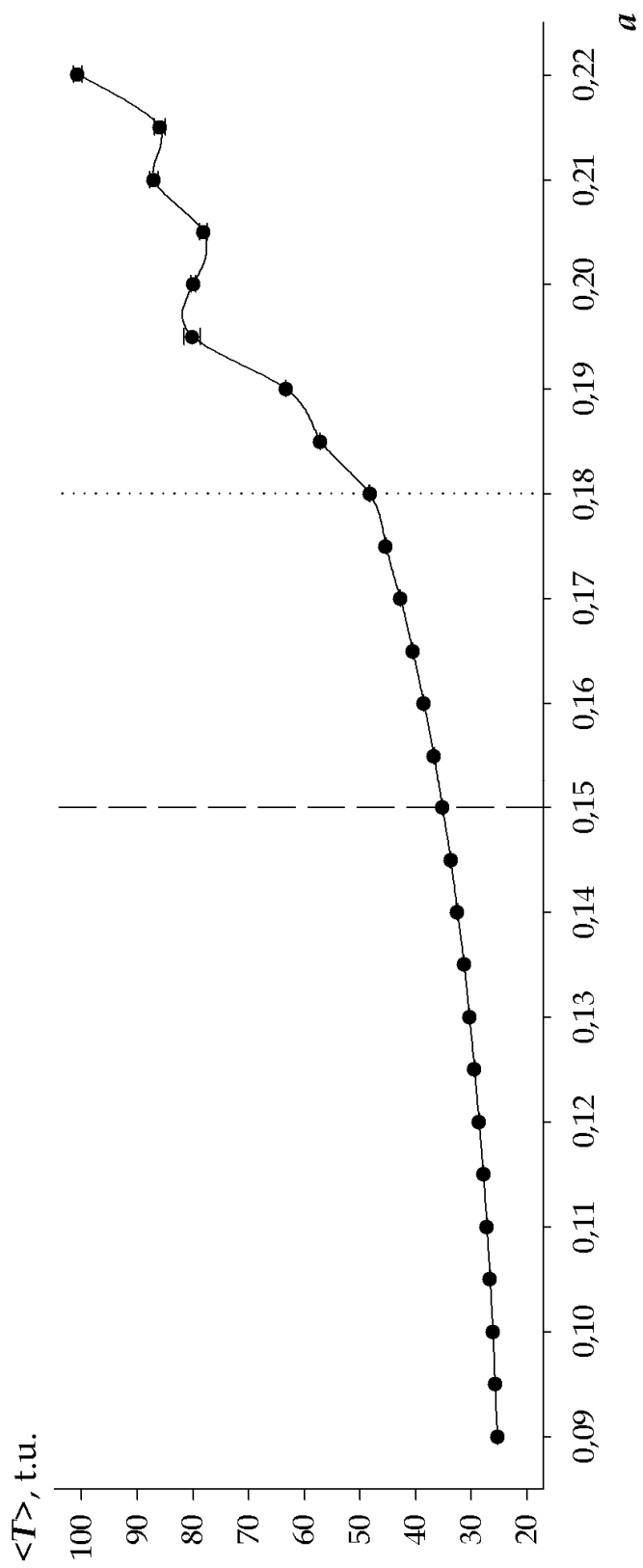

Fig. 2. Averaged ECG quasi-period (conventional units) as a function of the excitation threshold (parameter $a$ in the Aliev-Panfilov model) for monomorphic and polymorphic tachycardias caused by a single autowave reverberator. $a=0.150$ corresponds to normal excitability, and $a=0.18036 \pm 0.00007$ corresponds to the bifurcation boundary. 

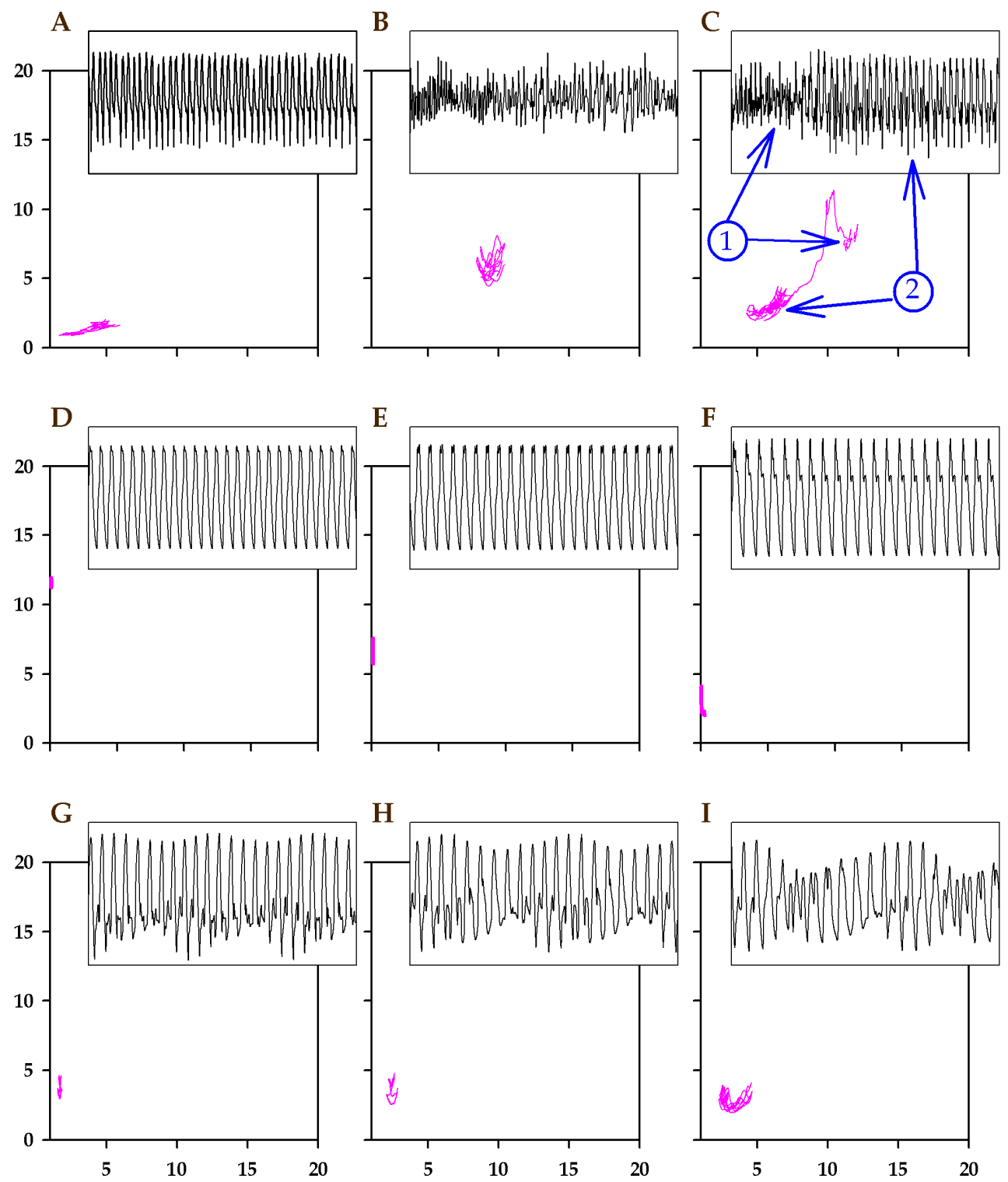

Fig. 3. The trajectories in the $\left(V_{1 i}, V_{2 i}\right)$ parameter plane for both "physiological" (upper row) and "numerical" ECGs (the others). Everywhere $V_{1 i}$, is abscissa and $V_{2 i}$ is ordinate. Each ECG fragment shown on the insert in the upper right corner corresponds to $5000 \mathrm{~ms}$. The middle row contains monomorphic ECGs, whereas lower row demonstrates polymorphic ones. For the middle row, $\mu_{1}$ is equal to 0.3 , and $a$ is equal to $0.15 ; 0.16 ; 0.17$ from (D) to $(\mathrm{F})$, respectively. For lower row, $\mu_{1}$ is equal to 1.3 , and $a$ is equal to $0.16 ; 0.17 ; 0.18$ from (G) to (I), respectively. 


\subsection{The same tachycardia observed from various standpoints}

Just as one is accustomed to watch that different dispositions of an object against a source of light produce sometimes very different shapes of the object shadow, the same should be expected for the ECG, if it is something like shadow, should it not? This hypothesis can be confirmed in a very simple experiment, the scheme and the results of which are presented in Fig 4. The numerical experiment demonstrates that one is able to obtain a desirable arrhythmic shape by choosing appropriate positions of the ECG recorders. The same experimental tachycardia arrived at the same time under the electrocardiographic guise of polymorphic arrhythmia similar to torsade de pointes in one lead and under the guise of nearly monomorphic arrhythmia in another lead. In this experiment, both electrocardiographic guises are engendered by a single autowave reverberator moving in meander manner, which is analogous as if human hands would concurrently produce a shadow of a rabbit on one wall and a shadow of a wolf on another. Could you manage it?

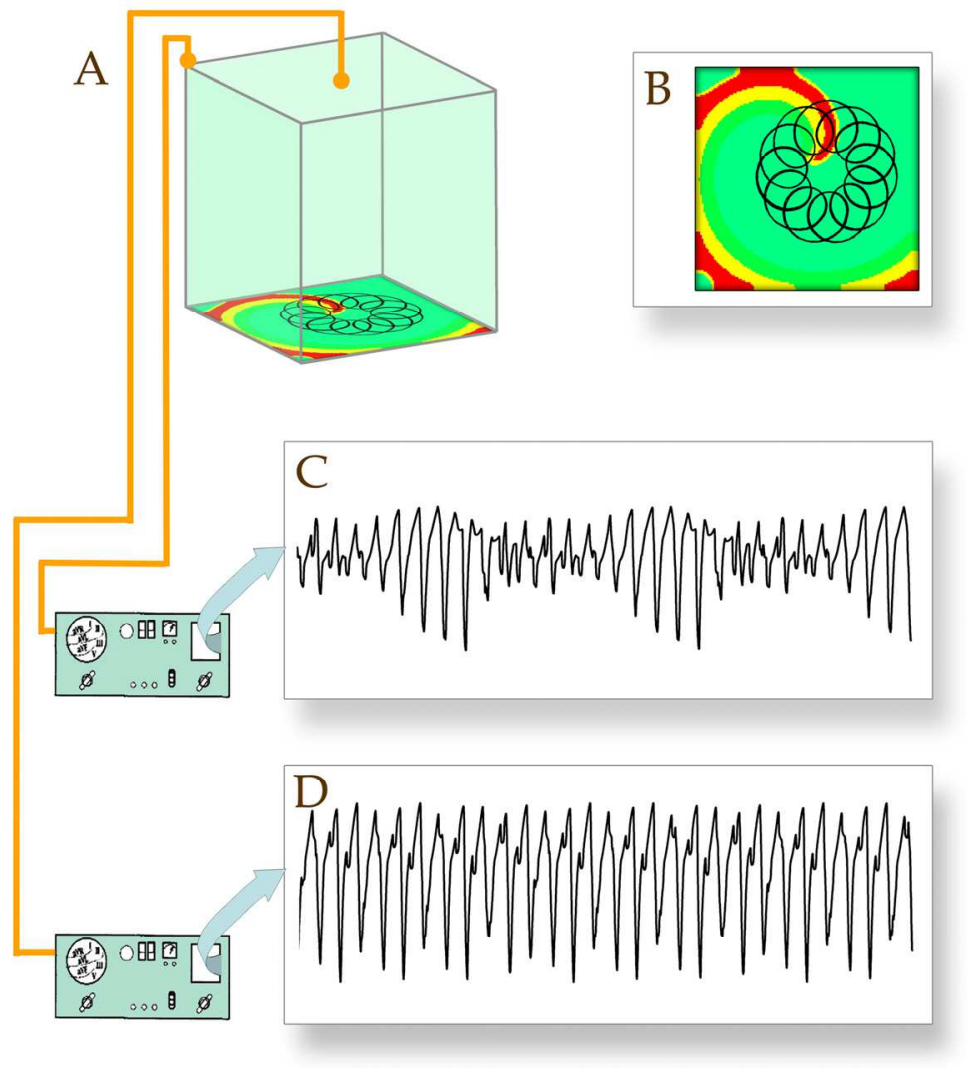

Fig. 4. Arrhythmic ECG shapes produced concurrently in different unipolar leads and engendered by a single autowave reverberator moving in meander manner during the same experimental tachycardia in model excitable medium. A - scheme of the numerical experiment. Insert: B - a trajectory of reverberator tip; C and D - ECGs, recorded over a corner and over the centre of the medium, respectively. Scale is the same for both ECGs. 
On the other hand, the similar ECG shapes can be caused by totally different arrhythmic sources. For instance, the following mechanisms are reported to be a cause of polymorphic tachycardia (Kukushkin et al., 1998): abnormal automaticity, focal triggered activity from after-depolarization, drift of a single re-entry, multiple re-entries or the excitation-wave overlap. Thus, it is doubtful that visual analysis of an ECG could in common case lead to the correct conclusion about the nature of the tachycardia, because an ECG seems to be just a "shadow" produced by one or another of the totally different arrhythmic sources.

\subsection{Is the degree of ECG polymorphism any informative?}

To tell the difference between more and less polymorphic ECGs, the term "degree of ECG polymorphism" was introduced (Kukushkin \& Medvinskii, 2004). According to primordial concept (Kukushkin et al., 1998), "arrhythmia was deemed monomorphic if the changes in the frequency and the amplitude of the pseudo-ECG during it did not exceed 15 and 30\%, respectively". Since being developed, the ANI-method has given a more detailed scale for measuring the degree of ECG polymorphism.

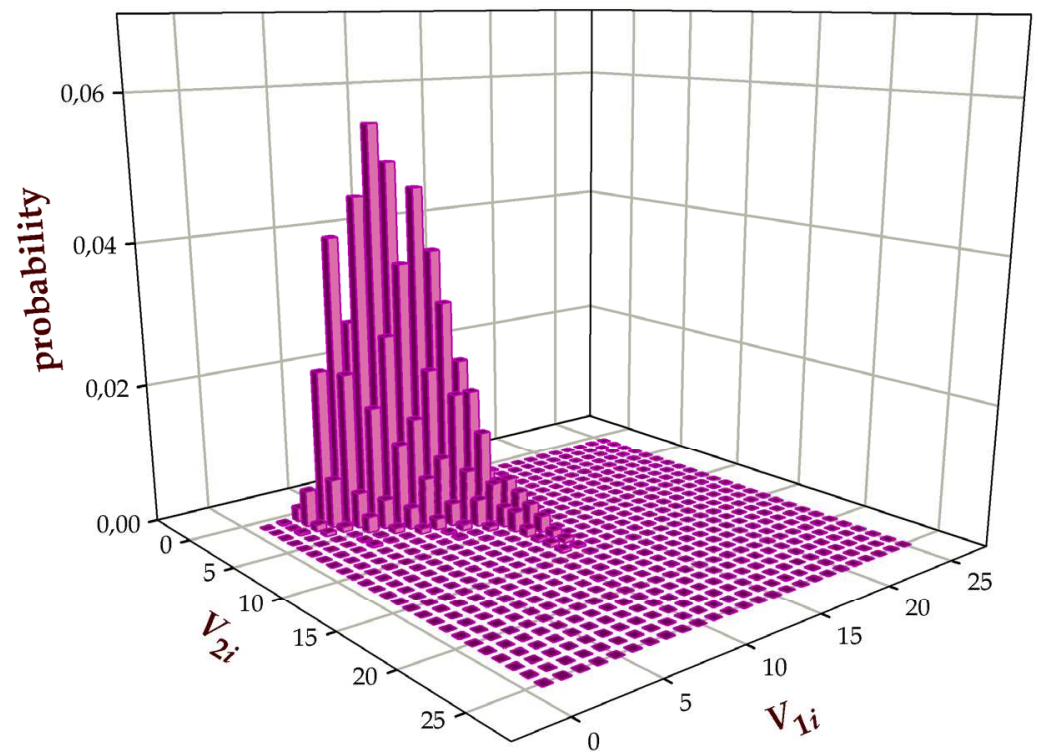

Fig. 5. Generalized histogram of "physiological ECGs", obtained from 12 physiological experiments and mapped onto the $\left(V_{1 i}, V_{2 i}\right)$ parameter plane.

One of exciting questions is: do different degrees of ECG polymorphism conform to different classes of polymorphic tachycardia? In other words, how many modes have the probability distribution of tachycardias with different degree of ECG polymorphism? In order to answer the question, more than 600 "physiological ECGs" were analysed with the use of the ANI2003. These were obtained from 12 experiments. The total duration of the ECGs was above 50 minutes and they put together more that twenty thousands arrhythmic cycles. The results are presented here for the first time (Fig. 5). One can see that the generalized histogram, which presents an estimation of the probability distribution of the "physiological ECGs" in the 
$\left(V_{1 i}, V_{2 i}\right)$ parameter plane, appears to be unimodal. Even if it is assumed to be polymodal, the different classes of tachycardia lie so compactly that they can hardly be segregated

The "physiological ECGs" were obtained (Moskalenko, 2009) in an experimental model study in vitro of ventricular tachycardia in the presence of different concentrations of lidocaine, a sodium channel blocker raising the membrane excitability threshold, which has been in broad clinical use as a local anesthetic and as an antiarrhythmic. Data were obtained with 10, 20, 3040,50 , and $100 \mu \mathrm{M}$. The incidence of arrhythmia with a certain degree of polymorphism was assessed by plotting the ECG distribution with respect to $V_{1 i}$. The efficacy of the antiarrhythmic influence can be evaluated by considering the probability of arrhythmia for which $V_{1}$ exceeds a certain value $V^{*}$ :

$$
P\left(V_{1}>V^{*}\right)=\left[1-F\left(V^{*}\right)\right]
$$

where $F\left(V_{1}\right)$ is the distribution function.

A control experiment demonstrated that the myocardial specimen itself, in the absence of lidocaine, remains quite stable over at least $8 \mathrm{~h}$, producing monomorphic kinds of tachycardia all the time. Though the histograms became somewhat broader with time, the modal value remained the same and the probabilities of polymorphic arrhythmia were practically zero. Only extrasystolia (3-7 oscillations) could be evoked at 40 or $50 \mu \mathrm{M}$ and no arrhythmia could be evoked at $100 \mu \mathrm{M}$, i.e., lidocaine produced a clear antiarrhythmic effect; these ECG data were not further processed. Note that in antiarrhythmic therapy, lidocaine has been commonly administered in doses creating a blood concentration over $50 \mu \mathrm{M}$. At $30 \mu \mathrm{M}$, lidocaine admitted some arrhythmia (19 cases of max. 3-s duration; in two cases spontaneous cessation was not registered). Upon visual inspection, these ECGs appeared more monomorphic than the reference ones. Administration of 10 and $20 \mu \mathrm{M}$ lidocaine did not change the duration of arrhythmias (recording up to $20 \mathrm{~s}$ ) and did not change appreciably the ECG patterns (in visual comparison with the initial reference).

However, in the histograms obtained upon ECG variability analysis (Fig. 6) one can see that $10 \mu \mathrm{M}$ and especially $20 \mu \mathrm{M}$ lidocaine increased the probability of polymorphic arrhythmia, whereas $30 \mu \mathrm{M}$ produced an opposite effect. When lidocaine was washed off at the final stage of the experiment, the histogram tended to revert to the initial form, but restoration was only partial, which can be explained by incomplete drug removal.

The results obtained with the induced-VT model confirm the usual "antiarrhythmic" effect of lidocaine at conventional "therapeutic doses," but reveal enhanced ECG polymorphism at lower drug concentrations. Since control specimens remained stable, this "paradoxical" influence should be attributed to the direct action of small doses of lidocaine, rather than to its poor efficacy at low levels against increasing inhomogeneity of myocardium. These experimental results are in nice accord with the earlier theoretical consideration (Efimov et al., 1995) of myocardial vortices in the Beeler-Reuter model as well as another theoretical study (Winfree, 1991) with a simpler FitzHugh-Nagumo model. In accordance with either theoretical work, intermediate levels of sodium conductance could produce drift of the excitation vortex, which corresponds to polymorphic VT. Thus, the findings for lidocaine reported here can likely be applied to other drugs of this class. Both theoretical works as well as this experimental work indicate that there is a nonlinear dependence of ECG polymorphism degree on myocardial excitability (and in this way on the concentration of sodium channel 
blockers): while myocardial excitability is reduced, the polymorphism degree is low first, then it increases and then becomes low again. This nonlinear dependence is in contradiction with the conventional medical conception according to which a monomorphic VT is much better than a polymorphic one. In other words, one should consider the therapeutic efficacy of sodium channel blockers as its pseudo-antiarrhythmic efficacy because the blockers at their socalled "therapeutically accepted levels" really make myocardium rather lifeless.

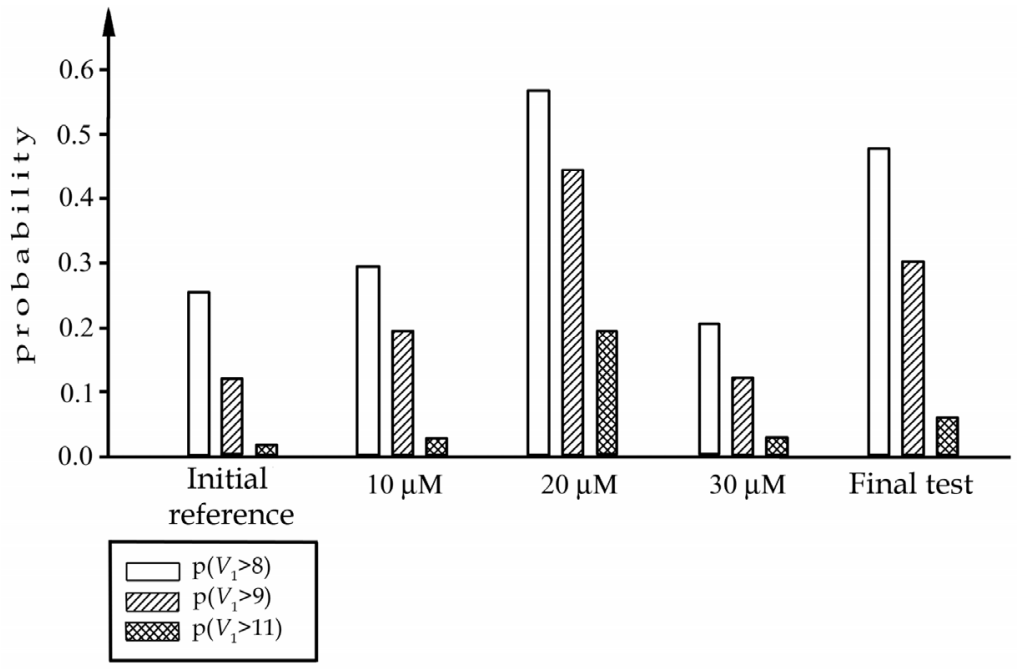

Fig. 6. The probability of appearance of the experimental ECG with different values of degree of polymorphism in the presence of different concentrations of lidocaine. Note again that the more value of $V_{1}$, the more degree of ECG polymorphism. The labels along the horizontal axis indicate the stage of experiment.

\section{Discussion and conclusions}

To disprove a theory, a single fact contradicting the theory is enough, but thousands facts are scanty to prove it. What new and good has this study been able to demonstrate?

First of all, the conclusion should be made, that monomorphic tachycardias appear to be rather mixed group of cardiac arrhythmias, which ought to be distinguished in very accurate manner in order to prescribe adequate treatment for cardiac disorders. The results presented here show that taking into account attractive resemblance of consecutive electrocardiographic shadows solely seems to be insufficient for good diagnostics.

Second, inasmuch as the phenomena of bifurcation memory in real myocardium are expected to be revealed, many cases of unsuccessful treatment of cardiac disturbances are likely to result from the phenomena. Within the scope of traditional electrocardiography, however, making diagnosis of bifurcation disorders in the heart seems to be doubtful.

Third, supposition about linear dependence of therapeutic effect on concentration of a medicament looks naive. Evidently, application of new techniques for monitoring "invisible" phase space of the heart would improve our capacity of using flexible and 
sophisticated schemata of medical treatment of cardiac diseases. Noble states: "There will probably therefore be no unique model that does everything at all levels. In any case, all models are only partial representations of reality. One of the first questions to ask of a model therefore is what questions does it answer best" (Noble, 2002b). So, appropriate collection of cardiac models is believed to improve both diagnostics and treatment.

In this investigation, the aim was also to assess the importance of polymorphic shape of the ECG in diagnostics of cardiac disorders. By painting a picture with metaphors and analogies, one create a visual image of one's concept; in doing so, one ensure that it sticks with one's prospect better than would a litany of industry- or science-specific terms. "Shadow play" appears to be a good suitable metaphor for understanding some problems of cardiology more deeply. As it was demonstrated in numerical study, there is no ground to believe, that degree of ECG polymorphism indicates severity of cardiac disease for certain. The only thing we can say without any doubt is that the electrical phenomena of the cardiac action attributed, in accordance with the most widely held current opinion, to different types of VT can be obtained in reality from a single patient concurrently. Thus, we should conclude that, in some cases, disgustful ugliness of consecutive electrocardiographic shadows during so-called polymorphic tachycardia is not likely to be as awful as the impression produced on a cardiologist by it. Besides, the customary classification of tachycardia should be improved.

This chapter has given an account of and the reasons for the widespread use of novel achievements of mathematics and physics for solving problems of cardiology. The results of this research support the idea that adequate diagnostics of cardiac disturbance should be grounded rather on observation of events on the "invisible" phase space of the heart than on raw sings in the electrocardiograms, which should be considered most likely to be a sort of intriguing "shadows" of the real cardiac action.

In this study, we have shown that the technique for ECG analysis referred to as ANI-2003 could provide cardiologists with a sensitive clinical tool for identifying life-threatening arrhythmias. The estimates derived from virtual ECGs in this study reveal some unexpected details of ventricular arrhythmia dynamics, which probably will be useful for diagnosis of cardiac rhythm disturbances. The data presented here pertain sometimes to "latent" polymorphism, revealed by the ANI-method while conventional ECG inspection did not reveal any important signs. The dependence of the trajectory location on ECG polymorphism defines a partial order in the $\left(V_{1 i}, V_{2 i}\right)$-space. The result is a new detailed quantitative description of polymorphic ECGs. Note that one cannot know the number of latent transitions in the ECG recorded during polymorphic ventricular tachycardia or ventricular fibrillation, but one can use the ANI-method to estimate it. The evidence from this study suggests that this method may prove useful in laboratory screening for new antiarrhythmics as well as in clinical testing to optimize the treatment regimen.

As concerns further investigations, studying influence of bifurcation memory phenomena on the cardiac action will lead to a better insight in the nature of cardiac diseases. Trying to understand nonlinear nature of cardiac arrhythmias, a cardiologist is expected to improve a cure for pathological tachycardia. Correct distinguishing between normal and pathological tachycardia seems to remain the most challenging problem for modern cardiology. Deeper comprehension of mechanisms of different sorts of both ventricular tachycardia and fibrillation is still required. Further work should explore also how re-entrant and focal 
arrhythmias could be distinguished by their ECGs. All these are good tasks for modern cardiovascular physics. Besides, further work needs to be done to establish whether the ANI-method is helpful for real clinical tasks.

\section{Acknowledgment}

Author is thankful for Dr. E. Shnol, Dr. Yu. Elkin, Dr. N. Wessel, Prof. A. Loskutov, and Prof. A. Ardashev for interesting discussion of important aspects of modern cardiovascular physics.

The partial support of Russian Foundation for Basic Research is acknowledged (the project 11-07-00519).

\section{References}

Aliev, R., Panfilov, A. (1996) A simple two-variable model of cardiac excitation. Chaos, Solutions \& Fractals, Vol.7, No.3, pp. 293-301, ISSN 0960-0779

Ataullakhanov, F., Lobanova, E., Morozova, O., Shnol', E., Ermakova, E., Butylin, A. \& Zaikin, A. (2007). Intricate regimes of propagation of an excitation and selforganization in the blood clotting model. Physics - Uspekhi, Vol.50, No.1, pp. 79-94, ISSN 1063-7869

Bushev, M. (1994). Synergetics: Chaos, Order, Self-Organization, World Scientific Pub Co Inc., ISBN: 978-9810212865

Crampin, E.J., Halstead, M., Hunter, P., Nielsen, P., Noble, D., Smith, N. \& Tawhai, M. (2004). Computational physiology and the physiome project, Experimental Physiology, Vol.89, No.1, pp. 1-26, ISSN 0958-0670

Efimov, I., Krinsky, V. \& Jalife, J. (1995). Chaos, Solutions \& Fractals, Dynamics of rotating vortices in the Beeler-Reuter model of cardiac tissue. Vol.5, No.3/4, pp. 513-526., ISSN 0960-0779

Elkin, Yu. \& Moskalenko, A. (2009). Basic mechanisms of cardiac arrhythmias, In: Clinical Arrhythmology (Russian), A.V. Ardashev, (Ed.), pp. 45-74, MedPraktika-M, ISBN 978-5-98803-198-7, Moscow

Feigin, M. \& Kagan, M. (2004). Emergencies as a manifestation of effect of bifurcation memory in controlled unstable systems. International Journal of Bifurcation and Chaos, Vol.14, No.7, pp. 2439-2447, ISSN 0218-1274

Kukushkin, N. \& Medvinskii, A. (2004) Ventricular tachycardias: and mechanisms. Vestnik aritmologii (in Russian), No.35, pp.49-55, ISSN 1561-8641

Kukushkin, N., Sidorov, V., Medvinskii, A., Romashko, D., Burashnikov, A., Baum, O., Sarancha, D., \& Starmer, F.C. (1998). Slow excitation wave and mechanisms of polymorphic ventricular tachycardia in an experimental model: Isolated walls of the right ventricles of the hearts of rabbit and ground squirrel. Biophysics, Vol.43, No.6, pp. 995-1010, ISSN 0006-3509

Kurian, T. \& Efimov I. (2010) Mechanisms of fibrillation: neurogenic or myogenic? Reentrant or focal? Multiple or single? Still puzzling after 160 years of inquiry. J Cardiovasc Electrophysiol., Vol.21, No.11, pp. 1274-5, ISSN: 1540-8167 
Kurian, T., Ambrosi, C., Hucker, W., Fedorov, V. \& Efimov I. (2010). Anatomy and Electrophysiology of the Human AV Node. Pacing Clin. Electrophysiol., Vol.33, No.6, pp. 754-62, ISSN: 0147-8389, 1540-8159

Loskutov, A. \& Mironyuk, O. (2007). Time series analysis of ECG: A possibility of the initial diagnostics. International Journal of Bifurcation and Chaos, Vol.17, No.10, pp. 37093713, ISSN 0218-1274

Loskutov, A., Shavarov, A., Dolgushina, E. \& Ardashev, A. (2009). Cardiac tissue as active media: Invariant characteristics of the theory of dynamical systems and heart rate variability. In: Clinical Arrhythmology (Russian), A.V. Ardashev, (Ed.), pp. 10851103, MedPraktika-M, ISBN 978-5-98803-198-7, Moscow

Medvinsky, A., Rusakov, A., Moskalenko, A., Fedorov, M., Panfilov, A. (2003). The study of autowave mechanisms of electrocardiogram variability during high-frequency arrhythmias: the result of mathematical modelling. Biophysics, Vol.48, No. 2, pp. 297-305, ISSN 0006-3509

Moskalenko, A. \& Elkin, Yu. (2007) Is monomorphic tachycardia indeed monomorphic? Biophysics, Vol.52, No.2, pp. 237-240, ISSN 0006-3509

Moskalenko, A. \& Elkin, Yu. (2009). The lacet: a new type of the spiral wave behaviour. Chaos, Solitons \& Fractals, Vol.40, No.1, pp. 426-431, ISSN 0960-0779

Moskalenko, A. (2009). Nonlinear effects of lidocaine on polymorphism of ventricular arrhythmias. Biophysics, Vol.54, No.1, pp. 47-50, ISSN 0006-3509

Moskalenko, A. (2010) Nonlinear and nonstationary ECG analysis of spontaneous transition from polymorphic to monomorphic arrhythmia in a mathematical model of cardiac tissue dynamics. Proceedings of the International Biosignal Processing Conference, pp. 084:1-4, Berlin, Germany, July 14-16, 2010

Moskalenko, A., Kukushkin, N., Starmer, C.F., Deev, A., Kukushkina, K. \& Medvinsky, A. (2001). Quantitative analysis of variability of the electrocardiograms typical of polymorphic arrhythmias. Biophysics, Vol.46, No.2, pp. 313-323, ISSN 0006-3509

Moskalenko, A., Rusakov, A. \& Elkin, Yu. (2008). A new technique of ECG analysis and its application to evaluation of disorders during ventricular tachycardia. Chaos, Solitons \& Fractals, Vol.36, No.1, pp. 66-72, ISSN 0960-0779

Noble, D. (2002a). Modelling the heart - from Genes to Cells to the Whole Organ. Science, Vol.295, pp. 1678-1682

Noble, D. (2002b). Modelling the heart: Insights, failures and progress. BioEssays, Vol.24, pp. 1156-1163, ISSN 0265-9247

Prigogine, I. (1980). From Being to Becoming: Time and Complexity in the Physical Sciences, W.H Freeman \& Co., ISBN: 978-0716711079, San Francisco.

Ramanathan, Ch., Ghanem, R.N., Jia, P., Ryu, K. \& Rudy, Y. (2004). Noninvasive electrocardiographic imaging for cardiac electrophysiology and arrythmia. Nature Medicine, Vol.10, pp. 422-428, ISSN 1078-8956

Sarancha, D., Medvinsky, A., Kukushkin, N., Sidorov, V., Romashko, D., Burashnikov, A., Moskalenko, A., Starmer, C.F. (1997). A system for the computer visualization of the propagation of excitation waves in the myocardium. Biophysics, Vol.42, No.2, pp. 491-496, ISSN 0006-3509

Titomir L.I. \& Kneppo P. (1994). Bioelectric and Biomagnetic Fields. Theory and Applications in Electrocardiology, CRC Press, ISBN 978-0849387005, Boca Raton. 
Vapnik, V. N. (1999) The Nature of Statistical Learning Theory (2nd edition), Springer, ISBN: 0387987800

Wessel, N., Malberg, H., Bauernschmitt, R. \& Kurths J. (2007). Nonlinear methods of cardiovascular physics and their clinic application. International Journal of Bifurcation and Chaos, Vol.17, No.10, pp. 3325-3371, ISSN 0218-1274

Winfree, A.T. (1991).Varieties of spiral wave behavior: An experimentalist's approach to the theory of excitable media. Chaos, Vol.1, No.3, pp.303-334, ISSN 1054-1500

Zhuchkova, E., Radnayev, B., Vysotsky, S. \& Loskutov, A. (2009). Suppression of turbulent dynamics in models of cardiac tissue by weak local excitations. In: Understanding Complex Systems, S.K. Dana, P.K. Roy, J. Kurths. (Eds.), pp. 89-105, Springer, Berlin 


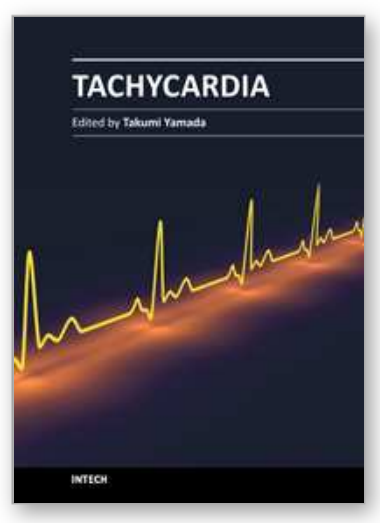

\author{
Tachycardia \\ Edited by Prof. Takumi Yamada
}

ISBN 978-953-51-0413-1

Hard cover, 202 pages

Publisher InTech

Published online 30, March, 2012

Published in print edition March, 2012

Heart rates are normally controlled by a natural pacemaker, the sinus node, and normal heart rhythm is called sinus rhythm. Tachycardia is defined as a faster heart rhythm than normal sinus rhythm. Tachycardias can cause symptoms such as palpitations, chest pain, shortness of breath and fatigue, which reduce the quality of life. Fast tachycardias can cause hemodynamic collapse and sudden cardiac death. The causes, mechanisms, and origins of tachycardias are various. The diagnosis of tachycardias is made by electrocardiograms and electrophysiological testing. Tachycardias can be managed and treated by pharmacological and nonpharmacological approaches. This book covers these concerns from basic and clinical points of view and will lead to a further understanding and improvement in the clinical outcomes of tachycardias.

\title{
How to reference
}

In order to correctly reference this scholarly work, feel free to copy and paste the following:

Andrey Moskalenko (2012). Tachycardia as "Shadow Play", Tachycardia, Prof. Takumi Yamada (Ed.), ISBN: 978-953-51-0413-1, InTech, Available from: http://www.intechopen.com/books/tachycardia/tachycardia-asshadow-play-

\section{INTECH}

open science | open minds

\section{InTech Europe}

University Campus STeP Ri Slavka Krautzeka 83/A 51000 Rijeka, Croatia Phone: +385 (51) 770447

Fax: +385 (51) 686166 www.intechopen.com

\section{InTech China}

Unit 405, Office Block, Hotel Equatorial Shanghai No.65, Yan An Road (West), Shanghai, 200040, China 中国上海市延安西路65号上海国际贵都大饭店办公楼405单元 Phone: +86-21-62489820

Fax: $+86-21-62489821$ 
(C) 2012 The Author(s). Licensee IntechOpen. This is an open access article distributed under the terms of the Creative Commons Attribution 3.0 License, which permits unrestricted use, distribution, and reproduction in any medium, provided the original work is properly cited. 